\title{
Biopolymer Composites for Slow Release to Manage Pimelea Poisoning in Cattle ${ }^{\dagger}$
}

\author{
Emilie Gauthier 1,* Diane Ouwerkerk ${ }^{2,3}$, Bronwyn Laycock ${ }^{1}$ and Mary Fletcher ${ }^{3}$ \\ 1 School of Chemical Engineering, University of Queensland, Brisbane, QLD 4072, Australia; \\ b.laycock@uq.edu.au \\ 2 Agri-Science Queensland, Department of Agriculture and Fisheries (DAF), Brisbane, QLD 4102, Australia; \\ diane.ouwerkerk@daf.qld.gov.au \\ 3 Queensland Alliance for Agriculture and Food Innovation (QAAFI), The University of Queensland, \\ Brisbane, QLD 4108, Australia; mary.fletcher@uq.edu.au \\ * Correspondence: e.gauthier@uq.edu.au \\ † Presented at the Third International Tropical Agriculture Conference (TROPAG 2019), Brisbane, Australia, \\ 11-13 November 2019.
}

Published: 13 February 2020

\begin{abstract}
Cattle grazing the pastures of inland Australia can be poisoned by ingestion of certain native Pimelea plant species, particularly Pimelea trichostachya and Pimelea simplex. The Pimelea toxin, simplexin, causes often fatal restriction of the pulmonary venules, with resultant heart impacts and characteristic fluid accumulation (oedema) of the jaw and brisket regions. In certain years heavy livestock losses can occur. Currently, there is no effective vaccine or antidote for Pimelea poisoning and the only management strategy is to reduce contact between toxic plants and susceptible stock, for beef producers to avoid potentially devastating poisoning events. Nevertheless, previous research has demonstrated that prolonged low dose feeding diminished the effect in animals. It was postulated that the animal exposed to prolonged low doses developed a mechanism for detoxifying simplexin, possibly through adaptation of the rumen microbial environment. The present study seeks to investigate the use of a biopolymer/toxin composite to foster toxin-degrading microbe populations. The objectives are to manufacture biopolymer composites based on biodegradable polylactic acid (PLA), polyhydroxyalkanoates (PHAs) and/or polycaprolactone (PCL), as toxin slow-release systems for the rumen that would have broad utility across a range of plant toxins and other beneficial rumen compounds. The poster covers the manufacturing, characterisation and performance of the biopolymers in a simulated rumen environment. Preliminary results of different biopolymers/composites containing Pimelea material and toxin extracts in an in vitro simulated rumen environment for up to 30 days are presented. It was found that the release rate could be tailored by choosing the right type of biopolymer.
\end{abstract}

Keywords: biodegradable biocomposite; slow release; Pimelea poisoning

Author Contributions: Material preparation, characterization and performance analysis: E.G.; Writing-original draft preparation, E.G.; writing-review and editing, E.G., D.O., M.T.F., B.L.; funding acquisition, M.T.F.; supervision, M.T.F, B.L. All authors have read and agreed to the published version of the manuscript.

Funding: This research was funded by Meat \& Livestock Australia, grant number B.GBP.0023.

Acknowledgments: The authors acknowledge the support provided by the Translational Polymer Research Group members and the Department of Agriculture and Fisheries technical staff.

Conflicts of Interest: The authors declare no conflict of interest. 
(C) 2020 by the authors. Licensee MDPI, Basel, Switzerland. This article is an open access article distributed under the terms and conditions of the Creative Commons Attribution (CC BY) license (http://creativecommons.org/licenses/by/4.0/). 